\title{
Badger Culling and Bovine TB in Cattle: A Re- Evaluation of Proactive Culling Benefit in the Randomized Badger Culling Trial
}

\author{
Thomas ES Langton* \\ Triton House, United Kingdom
}

Submission: May 10, 2019; Published: May 23, 2019

*Corresponding author: Thomas ES Langton, Triton House, Bramfield, Halesworth, Suffolk IP199AE, United Kingdom

Abstract

Aspects of design and analysis of the Randomized Badger Culling Trial in England are considered with respect to the relationship between proactive badger culling and incidence of new bovine tuberculosis cattle herd breakdowns. Assumptions made by the Independent Scientific Group report in 2007 on the RBCT experiment including its design are reviewed. Independent re-evaluation of RBCT data does not show a statistically significant relationship between proactive badger culling and new herd breakdown. Better understanding of the pathogenesis of the disease since the RBCT experiment and an alternative statistical approach challenges the methods, analyses and conclusions of the effects of proactive culling in RBCT. Hence the validity of its current application in large scale badger culls in England since 2013 is questioned.

\section{Introduction}

Bovine tuberculosis (bTB) is a bacterial disease of cattle, caused by the bacterium Mycobacterium bovis, which also affects other wild and domestic mammals. It has returned significantly to west and central England and is still spreading since its extensive removal in the 1960s. The scientific evidence base, principally the Randomized Badger Culling Trial (RBCT) experiment 1998-2005 together with the economics and the ethical aspects of massculling of the European badger Meles meles is highly contested by many stakeholders [1]. This is partly because the current disease intervention strategy does not seek just to remove bTB infected badgers but to kill a majority of animals in a badger population over a very large area including well away from cattle herds, in an attempt to influence any badger-to-cattle transmission. The level of certainty needed to undertake the mass-culling of a traditionally persecuted and legally protected species in order to enable a veterinary intervention of uncertain contribution, forms the focus of this paper.

Across the European range of the badger, many attempts have been made in studies to try to distinguish between cattle to cattle transfer of bTB, bTB pathogen pollution of the environment from dung and faecal slurry spreading and infection from other animal hosts, including domestic animals and wildlife. These studies estimate the relative contribution to infection and spread of bTB, according to local factors. These include transmission within complex multi-host livestock assemblages [2-4], involvement of non-bovines such as sheep, goats and pigs and wild mammals such as wild boar (Sus scrofa), roe deer (Capreolus capreolus), red deer (Cervus elaphus) and red fox (Vulpes vulpes).

Badger populations in parts of Britain and Ireland are some of the highest reported within its range [5]. Despite the badger being described occasionally as a bTB maintenance host or reservoir there is no confirmed evidence of this [6]. However, it is not the intention in this paper to review the many aspects of detailed local studies of infected farmland or countryside across Europe. It focuses very specifically on the validity of a single and large-scale badger removal intervention in England in order to examine the strength of science behind it and its likely potential contribution to disease control. The context of the RBCT badger culling experiment design in the mid to late 1990s was that badger transmission was circumstantially deemed to be the major factor associated with BTB transmission. Few restrictions were put on the very extensive movement of cattle in England and consequently the bTB epidemic in cattle has spread progressively wider across the country since then.

Government policy and approaches to allow extended depletion of badger populations [7-10] was based on original scientific data in respect of whether the intensive proactive culling of badgers can contribute to any subsequent measurable reduction in bTB cattle herd breakdown. Concerns regarding this strategy include the approval of extended culling (supplementary 
culling) to retain badgers at low levels after an initial four-year period of badger depletion (intensive culling). Intensive culling was authorized

a. To maintain low badger numbers in 'High Risk Areas', and

b. To cull high proportions of badgers when disease has been recently introduced by cattle movements and has the potential to spread to badgers and cattle locally within a 'Low Risk Area' covering much of the East of England. Supplementary and Low Risk Area culling extensions are based partly upon findings since the 2011 policy was confirmed and have been designed in an environment where their contribution to disease control is unknown or scientifically unproven. Recent considerations have been given to reinstating periodic, as opposed to continuous badger culling noting that "the greatest benefits from repeated widespread culling occur in the first two years after culling is stopped" [6].

The RBCT was the basis of the subsequent UK government bTB control policy and strategy. This experiment culled badgers repeatedly to remove up to an estimated $80 \%$ or more of the population within defined areas. It culled badgers in places where bTB herd breakdown was recorded as most intensively increasing, as revealed by bTB cattle testing and a single category of bTB test reactors known as 'confirmed' reactors [11]. The RBCT recorded the impact of badger culling on the level of bTB New Herd Breakdowns (NHB) in controlled $100 \mathrm{~km}^{2}$ geographic areas with the proactive removal of as many badgers as possible by the shooting of cage-trapped individuals. This was done within an intensive period of culling of under two week's duration, following pre-baiting of trapping areas with food bait.

The reactive culling method within similar sized areas aimed to remove all badger social groups having access to a bTB breakdown farm and so was a patchier or localized treatment. Reactive culling was suspended by the government in 2003 due to reporting by the ISG that it was giving rise to an estimated increase in bTB NHB in the reactive trial areas [11]. The RBCT produced data from ten 'triplet' (proactive, reactive, control) areas in west England. Each triplet was chosen so that the three areas were as close to each other geographically and as similar as possible in terms of explanatory variables that were not explicitly measured in the study, e.g. weather patterns and soil type. The RBCT outcome variable measured was the number of NHB, as indicated by single intradermal comparative cervical tuberculin test (SICCT) detection of bTB and post-mortem examination to categorize 'confirmed' reactors. The RBCT experimental design and its statistical analysis of proactive badger culling $[12,13]$ was carried out at a time when the Krebs Review expressed a view that while recently infected cattle do excrete $M$. bovis, that cattleto-cattle transmission is not common [14]. In view of recent data in multiple situations this is clearly incorrect.

In this reappraisal, and in the acknowledgement of new scientific and field-based evidence, we can critically consider aspects of proactive badger culling in the RBCT. Specifically, aspects of the following areas of these trials are considered:

i. Control of a potentially significant confounding variable; uneven immigration of bTB infected cattle into trial areas before and during the experiment;

ii. The non-blindness of the trials;

iii. A summary of the previous analysis of the RBCT data;

iv. An alternative analysis of the main results for proactive culling.

Finally, this paper discusses why the alternative analysis provided is preferred to that chosen and why it better fits with historical understanding, recent investigations and discoveries in bTB pathogenesis since the RBCT experiment was conceived and commenced over twenty years ago.

Control of a Potentially Significant Confounding Variable; uneven bTB Infected Cattle Immigration to trial areas

It is important to identify aspects of a randomized study that might lead to uneven balance between paired treatment and control areas. The final model used in the RBCT analysis controlled for two variables only: cattle herd density and the different number of NHB in trial areas in the years prior to the experiment starting. One potential uncontrolled variable identified here is the disparity in SICCT test frequency in areas surrounding the trial areas before and during the experiment. In the 1990s, districts of England with bTB outbreaks were placed in SICCT testing cycles of between one and four years, according to the level of NHB locally and giving rise to a variable pattern of testing frequency [11,15]. Testing frequency changed throughout the three years prior to and during the RBCT experiment; a period of around ten years. The situation in the three years before the experiment began and during its first five years is unclear as generally areas with parishes on fouryear bTB testing, moved to more frequent testing. The potential disparity between cull and control and its impact of differential disease burden on stock moved around during the trials may be far more exacerbated than can be deduced by considering the 2005 parish testing frequency-patterns (Figure 1) [15] alone.

For the proactive vs control paired comparisons in the RBCT, in 2005 [13], around half have one member of the pair more heavily surrounded by other high intensity breakdown areas (often other RBCT cull triplet members) than its other paired area. Thus, there is considerable chance of uneven influence from stock of unpredictable disease burden entering trial areas. In the Fig.1 map referred to above, in around half of the cases, one of the cullcontrol pairs is closer to an area with parishes on SICCT testing at two-year intervals, but there was no consistent variation, creating uncertainty and a potential source for erratic influence upon the experiment. This issue may not have been considered at the time because of the Krebs Review statement that cattle-to-cattle transmission from recently infected cattle was not common. 


\section{The Non-Blindness of the Trials}

The experimental blinding aspect of the RBCT field trial is a concerning factor. Whether or not badgers are being cage-trapped and shot in study areas is hard or impossible to conceal due to the extensive administrative and physical procedures involving many people and in areas with public access. Other small-scale and non-blinded badger culling trials prior to the RBCT contributed to the call for a more rigorous experimental approach to address bTB research issues [14]. However, the lack of blinding in order to remove unconscious bias remained a major shortfall in the overall RBCT experimental design. When badgers are being culled and herds are under closer official scrutiny, aspects of herd management such as on-farm biosecurity effort and risky trading behavior in respect of bTB infection may change. This includes levels of unregulated and unlawful stock movement. It would only take a slight shift in management behavior to obtain the small difference in bTB breakdown rate and that might otherwise be erroneously attributed to disease transmission by other vectors. The direction of change might not be clear, and it should be noted that in the RBCT experiment the direct response to culling badgers was for bTB to rise almost as often as it fell across the study.

Table 1: Vet Net data for the number of herds contracting BTB in the Control and Culled areas and the within triplet (paired comparison) difference. The table also gives the number of NHBs (I3) in the previous three years before the trial began (for culled and control areas) and the number of baseline herds $\mathrm{B}$ at the start of the trial [11].

\begin{tabular}{|c|c|c|c|c|c|}
\hline Triplet & Culled & Control & Culled - Control & B Culled, Control & I3 \\
\hline A & 40 & 67 & -27 & 71,89 & 153,133 \\
\hline B & 98 & 70 & 28 & 107,173 & 13,27 \\
\hline C & 34 & 98 & -64 & 98,108 & 28,30 \\
\hline D & 39 & 49 & -10 & 116,101 & 25,28 \\
\hline E & 42 & 67 & -25 & 142,190 & 12,34 \\
\hline F & 16 & 64 & 29 & 245,131 & 26,15 \\
\hline G & 83 & 54 & -6 & 66,129 & 23,22 \\
\hline H & 36 & 42 & 7 & 107,98 & 30,19 \\
\hline I & 38 & 31 & 6 & 116,124 & 25,18 \\
\hline J & 46 & 40 & & & 2 \\
\hline
\end{tabular}

Donnelly [13] fitted log-linear models to the number of NHBs in the second and third columns of Table 1 . These authors assumed that the bTB data had a Poisson distribution given the values of the explanatory variables. A simple model is that

$$
\log (\mu)=\beta 0+T R I P+T R E A T
$$

where $\mu$ is the mean level of NHBs, TRIP is a factor indicating which triplet (A to J) the observation was in and TREAT is a treatment factor with two levels (culled and control). This model is essentially the log-linear model equivalent of the paired t-test described above. It generates a similar $p$-value for the null hypothesis that the TREAT effect is zero $(\mathrm{p}=0.25)$. An approximate $95 \%$ confidence interval for the expected difference in the effect of culling on NHB as a percentage of the expected NHB for control areas is $(-42.9,15.2)$. In other words, the model suggests that

\section{Previous Analysis of the RBCT proactive culling data}

Table 1 summarizes NHB from the period of the initial proactive cull to the end of the trial. For each triplet, the "Culled Control" column shows the difference in NHB between the culled and control areas (not including the $2.0 \mathrm{~km}$ wide buffer zone around the main treatment areas). A negative value indicates that there were more NHB in the control area and a positive value indicates that there were more NHB in the culled areas. These data show that culling produced fewer NHBs in 6 out of the 10 paired comparisons, so, an initial view is that badger culling appeared to result in reducing NHBs in six areas but increasing it in four areas. The standard analysis for the data presented in Table 1 is to consider differences within paired comparisons. Whilst these data are counts and hence not continuous, the counts are sufficiently high for a t-test assuming Normality, to give a reasonably accurate $\mathrm{p}$-value. When the t-test is carried out on these data, it gives a $\mathrm{p}$-value of 0.28 , suggesting no evidence for a difference between control and culled treatments. The correctness of the p-value was confirmed by a non-parametric randomization test based on 10,000 randomizations. This gave exactly the same p-value of 0.28 . the effect of culling could be positive or negative. Donnelly [13] fitted a similar model to (1) but included two further explanatory variables to help explain the variation in NHBs. Their model was

$$
\log (\mu)=\beta_{0}+\beta_{1} \log \left(I_{3}\right)+\beta_{2} \log (B)+\text { TRIP }+ \text { TREAT --- }
$$

where $\log (\mathrm{B})$, the $\log$ of number of baseline herds (i.e. the density of herds in each area) and $\log \left(I_{3}\right)$, is the log of the number of NHBs in the 3 years before culling began. These authors also added 0.5 to the values of $I_{3}$ and $B$ before the logs were taken, because they said this would minimize bias in the covariates. The $95 \%$ confidence interval for this model was $(-32.7,-12.4)$ with a mean of -23.2. The $\mathrm{p}$-value for the culling effect was $\mathrm{p}<0.001$. The confidence interval suggests that the whole of the range of plausible values for the mean effect of culling is negative. In other words, that badger culling reduced the mean NHB level. 


\section{An Alternative Way to Model NHB}

For the analysis of results from the RBCT trial to be robust, we would expect other plausible models to produce the same conclusions. The approach taken was not simply to search for one that contradicted the existing analysis in some kind of targeted attempt to undermine it, but to start by looking at approaches most similar and logical to that taken by the RBCT. One such model is defined below and was the first alternative approach attempted. This used some of the information from Table S1 in Donnelly [13]. This is reproduced in Table 2, below. The first column of Table 2 gives the number of years of observation (NY) of each of the triplets. This is important because NY varied from 3.40 (triplet D) to 7.88 (triplet B). This information was used to create a standardized variable NHB*, which was the number of new herd breakdowns per baseline herd year at risk. Using a similar calculation to that done in Table 6 of Donnelly et al. (2006, supplementary material) we get

$$
N H B^{*}=\frac{N H B}{\left(N Y^{*} B\right)}
$$

A better way might be to use the number of baseline herd information rather than to simply use it as an explanatory variable (as done in model 2). A simple way to model NHB* is to assume that it has a Gaussian distribution and to model it by the standard linear model

$$
N H B^{*}=\beta_{0}+\beta_{1} I_{3}+\text { TREAT }+ \text { TRIP }+ \text { error }---
$$

Table 2: Number of years of observation of each triple, number of herd years at risk, and new herd breakdowns per herd year at risk for control and culled areas.

\begin{tabular}{|c|c|c|c|c|}
\hline Triplet & $\begin{array}{c}\text { Number of years of } \\
\text { observation (NY) }\end{array}$ & $\begin{array}{c}\text { Breakdowns per herd years } \\
\text { at risk (NHB*) for Culled } \\
\text { areas }\end{array}$ & $\begin{array}{c}\text { Breakdowns per herd years } \\
\text { at risk (NHB*) for Control } \\
\text { areas }\end{array}$ & Culled - Control \\
\hline A & 6.74 & 8.36 & 11.17 & -2.81 \\
\hline B & 7.88 & 8.13 & 6.68 & -3.45 \\
\hline C & 6.90 & 4.61 & 8.21 & -1.64 \\
\hline D & 3.40 & 11.70 & 13.34 & -4.78 \\
\hline E & 6.30 & 5.75 & 10.53 & -3.79 \\
\hline F & 5.92 & 1.90 & 5.69 & -1.31 \\
\hline G & 6.61 & 6.04 & 7.35 & 3.91 \\
\hline I & 5.63 & 9.69 & 5.78 & 1.02 \\
\hline J & 3.80 & 9.35 & 8.32 & \\
\hline
\end{tabular}

where the error is assumed to be Normal with constant variance. The plots of studentized residuals against fitted values and the $\mathrm{Q}-\mathrm{Q}$ plot of studentized residuals shown in Figure 1, suggest that the assumptions behind this model are reasonable. Apart from the number of baseline herds, this model includes the same explanatory variables that are in Donnelly's model (2). When fitting this model, we get approximate 95\% confidence intervals for the effect of culling of $(-2.8,0.7)$ for the Vet Net data. That is, that culling changes the expected number of NHBs by between -2.8 and 0.7 . Thus, from the confidence interval, this model does not suggest that proactive culling necessarily reduces the number of NHBs - it may increase it or decrease it. The data are taken from Table 1 in Donnelly (2007) and shown in Table 2. Which allows this analysis to be replicated.
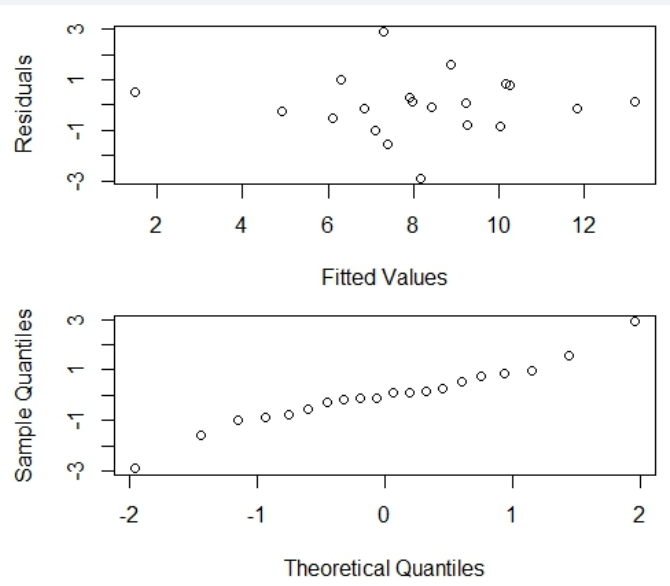

Figure 1: Residual plots for the alternative model (3). The top plot shows studentized residuals against fitted values. The bottom plot is a Normal $Q-Q$ plot of the studentized residuals. Both plots suggest that model (3) is a reasonable representation of the data. 


\section{Discussion}

There are three layers to the problems with the RBCT analysis and its use to justify proactive badger culls. These are:

a. The confounding variable caused by unknown yet almost certainly uneven disease immigration between cull and control trial areas due to cattle movements;

b. The fact that the study was non-blind and so open to herd manager behavior bias; and

c. The non-statistical significance of the results when modelled in a slightly different way.

The confounding variable caused by unknown yet likely to be uneven disease immigration between cull and control trial areas due to cattle movements

This could be an important confounder because SICCT testing frequency influences local bTB prevalence rates and hence infection risk. Cows are very regularly traded and moved around the countryside, often in small numbers over small distances. Different distance from trial areas to areas with differing testing, including those between cull and control pairs may give rise to the relatively modest differences in NHB of the level observed in the RBCT experiment. The RBCT raw data shows that the total difference between proactive cull areas and their controls was under two breakdowns per year on average, in what was already a minimal experimental sample size of 10 paired comparisons. An unstable experimental environment existed due to the uneven distance of trial areas from areas supplying cattle under different SICCT testing frequency. The frequency changed before and during the trials. Introducing cows with uneven disease exposure to cull and control is a confounding variable with no measurement. It needed to be addressed just as much as pre-trial NHB variation was considered vital. This factor could have been checked through recording the origin of stock at the time of the experiment in the study area.

\section{Study was Non-Blind and so Open to Herd Manager Behavior Bias}

Changes in stock management behavior once badger culling has started on farms were not studied. More frequent visits by officials and scrutiny by administrators and field workers may induce changes that differ between cull and control and between paired comparisons in an inconsistent way. The blinding of trials is increasingly recognized as essential.

\section{The Non-Statistical Significance of the Results when Modelled in a Slightly Different Way}

Ultimately, even when considering the historic RBCT data as it has been presented, which of the models 2 (RBCT model), or 3 (the current model) should be used to draw conclusions from? The first says that culling may reduce NHB and the second says that it might not. This is not a simple question to answer, yet there are major disease-control, animal welfare, environmental, legal and economic and stakeholder welfare considerations based on the findings. All that can be said is that the two ways to model the data do not come to the same conclusion. The conclusions of the frequentist RBCT analysis should be robust to the way it is analyzed - so long as the methods used are sensible and appropriate. The alternative formulation of the analysis model in (3) is entirely plausible because it uses a similar variable (new herd breakdowns per year at risk) to that presented in the original RBCT report.

\section{Why the Alternative Analysis makes sense from Current Understanding of Pathogenesis}

In addition to the comments on confounding variables and experimental non-blinding it is important to explain why the alternative analysis makes epidemiological sense due to a clearer understanding of pathogenesis of bovine tuberculosis since the RBCT experiment was designed. A very detailed summary of bTB experimentation and observation was published more than 70 years ago [16]. This was at a time when infection and postmortem of guinea pigs Cavia porcellus using samples taken from cows was used to detect and to follow bTB disease transmission in cattle. Aspects of bTB pathogenesis that were applied in the removal of the disease in the 1960s appear in policy terms to be progressively disregarded from the 1970s onwards. A new view developed from that time in cattle veterinary circles that only cows with visible lesions on slaughter were capable of passing on infection and routinely investigated [17]. This is clearly incorrect as shown by subsequent data.

However, unlike human TB, small tuberculous lesions may remain open in bovines making onward transmission via the potential routes of aerosol transfer, parturition secretions, saliva or faecal infection possible $[16,18]$. bTB microlesions are often too small to be visible in the abattoir and the very low density of bacteria from inactive infections are below the level required for reliable laboratory culture yet they may still be infectious. Calves and cows in their early years of life with subclinical infection may both be infectious for short periods and be hard or impossible to detect with the SICCT test because of undeveloped immune responses. Such factors combined with long lead times for clinical symptoms to show lead to unpredictable emergence of breakdown from apparently bTB-free stock even after generations, giving the appearance of a cryptic external infection source, as opposed to latent disease. The view on low infectiousness of young cows does not consider transfer of bTB from undetected mother to calf at birth with slaughter of mother prior to SICCT detection, or early post-partum infection from other undetected source adults including from pooled unpasteurized milk feed. It has always been known that some categories of infected cattle may be hard or impossible to detect with the SICCT test and that movement of them will introduce disease. Views of bTB transmission between cattle as a slow and uncommon event may explain the lack of attention, in the RBCT experiment's design, to control the variable rate of spread of bTB through inter-herd cattle movement that is extensive and characterizes the UK cattle supply system [19]. 
Risk of bTB transmission comes from cows that have contracted bTB and are incubating the disease or have it present in a latent or dormant state $[16,20]$ but are negative in SICCT.

Levels of within-herd bTB persistence has been recorded in recent years, with approximately $38 \%$ of herds certified as officially TB free at the resolving of a breakdown going on to experience a recurrent incident within 24 months [21]. This may be linked to failure to detect residual Myobacterium infection sufficiently to prevent sustained infection that would be avoidable using a test that is not dependent upon immune response. An example is bacteriophage amplification testing to directly identify live Mycobacterium avium subsp. Paratuberculosis infection as opposed to immune response tests for paratuberculosis [22]. Exceptional and conditional use of Actiphage-testing of blood and milk may now be applied for and use of additional tests to SICCT has increased in recent years. Other tests being more widely used include gamma interferon (IFN- $\gamma$ ) testing, the IDEXX ELISA antibody detection assay and PCR DNA amplification tests [23].

Understanding of the efficacy of the SICCT, which is the approved stand-alone herd test for bTB infection in living cattle is long established [20]. Standard SICCT test sensitivity was reported at 68-95\% around the time of the RBCT design [24] but more recently only $40-80 \%$ of infected animals [20]. Other recent analyses have found a similar range [25] and so the probability of two or more SICCT tests leaving one or more infected animals is relatively high. Infected animals are left in the herd, leading to perpetuation of cattle-cattle transmission for those not taking heed of these findings or those of Francis [16]. The specificity levels of the SICCT test are not doubted and 'unconfirmed' reactors are now no longer considered just to be 'false positives', especially in endemic TB areas [27]. When considering ultra severe cut off (reactors and indeterminate reactors) of SICCT, it has been considered 99.87 percent specific [28].

More \& McGrath [29] suggested that misclassification of nonpositive reactors may be least with the use of 'confirmed' reactors. But the status of the so called 'unconfirmed' SICCT test reactors is a matter where understanding has improved. The RBCT found a statistically significant relationship between proactive badger culling and NHB using visible tuberculosis lesions and culture test positive reactor cows ('confirmed' breakdowns). It found no statistically significant relationship when including reactors classed as 'unconfirmed' breakdowns, where physical examination and culturing failed to indicate bTB, yet the cow's immune system reacted, indicating bTB history. Even 'indeterminate' reactors, where SICCT reaction to skin swelling is below the size for compulsory slaughter have been shown to indicate bTB infection $[16,28]$. This adds further to the doubt as to the selection of 'confirmed' breakdown only as any constant measure of arrival of new infection and NHB. It also contradicts any assumption that there is no bTB 'carrier state' in cattle. 'Confirmed' breakdowns only may be an erratic way of recording NHB compared with the available alternatives and this is yet another aspect of the RBCT that indicates uncertainty. Many of the reference models in published literature may also need re-running using the lower range of likely sensitivity of the SICCT test, now that it is better understood.

The clarification of these epidemiological processes all points to a far greater burden of disease remaining in herds that have been declared bTB-free. Not only do they cast doubt over models that assume the SICCT test has high sensitivity but can help explain why the view that an external vector was significant when cattle to cattle infection could be the total or near total source of infection. Applied use of the RBCT has continued since 2007 with post-cull NHB data being used to look at medium term NHB trends in the RBCT proactively culled badger areas [15,30,31]. Further analysis of NHB in the years after the initial RBCT culls and over longer periods has now been conducted $[32,33]$ with new approaches to continue depleting badger populations after an initial four-year period of proactive culling. Pilot culls based upon RBCT methods were started in two areas in 2013 one in Gloucestershire and one in Somerset as a part of the 2011 policy to bring about bTB control in England [10]. However, in respect of monitoring any outcomes, conditions within two study areas are different and there are no standard controls. There is use of additional bTB testing methods in these areas and it is not possible to indicate how any change in prevalence or incidence of bTB relates to the removal of badgers. This makes the RBCT and the ISG findings even more important as they are being treated as hard evidence against which to extend new badger culling approaches that are more simplified and distant from the published science and any quantifiable measure.

Whether there is any real reduction in bTB NHB incidence following proactive badger culling is far from certain. Whether cattle contract bTB from badgers at all in farm conditions (or more frequently by cattle from badgers than from other multiple sources of on-farm bTB contamination) also remains unclear and would benefit from further investigation. The 'King Review' [34] pointed out the weak statistical strength of the badger bTB perturbation effect, questioning the validity of the badger bTB perturbation hypothesis [35] and these may also benefit from reassessment. The only molecular evidence ever demonstrated using state of the art tools is from cattle to badger.

\section{Conclusion}

Problems that are apparent now, largely in hindsight in the RBCT design, may have been carried forward into very many subsequent derivative papers. This reappraisal finds that the conclusion of Godfray [36] that "proactive culling, as conducted in the trial [RBCT], resulted in a relative reduction in new confirmed cattle herd breakdowns inside culling areas", does not address the strength of the findings in the light of alternative and new information since the RBCT and alternative statistical approaches. Those findings, had the authors looked in any depth at the experiment and subsequent publications would have been better classified under a different category used; "substantial uncertainty exists that could affect outcomes." Exceptions made for licenses to be issued to allow very extensive badger culling 
under the Protection of Badgers Act (1992) 'for the purpose of preventing the spread of disease' are not based upon robust scientific evidence as may be required to justify derogations from the law. Notably this uncertainty may apply to those licenses issued for badger culling since 2013 in England, elsewhere in the UK and Ireland and elsewhere where there has been reliance on the findings of the RBCT and papers derived in part from its data or assumption [36].

\section{Acknowledgements}

Four external anonymous reviewers are thanked for extensive comments and suggestions, resulting in a range of useful additions, corrections and clarifications to previous manuscripts. Five additional advisors on statistical methods, bTB testing and research analysis are thanked for considerable advice and assistance during the preparation period.

\section{References}

1. McCulloch SP, Reiss (2017) M.J. Bovine tuberculosis and badger control in Britain: Science, policy and politics. Journal of Agricultural and Environmental Ethics 30(4): 469-484.

2. Malika Bouchez-Zacria M, Courcoul A, Durand B (2018) The Distribution of Bovine Tuberculosis in Cattle Farms Is Linked to Cattle Trade and Badger-Mediated Contact Networks in South-Western France, 2007 2015. Front Vet Sci 5: 173.

3. Guta S, Casal J, Napp S, Saez JL, Garcia-Saenz A, et al. (2014) Epidemiological investigation of bovine tuberculosis herd breakdowns in Spain 2009/2011. Plosone 9: e104383.

4. Cowie CE, Beck BB, Gortazar C, Vicente J, Hutchings MR, et al. (2014) Risk factors for the detected presence of Mycobacterium bovis in cattle in south central Spain. Eur J Wildl Res 60: 113-123.

5. Roper T (2010) Badger, Collins New Naturalist, London, UK.

6. Godfray HCJ, Donnelly CA, Hewinson RG, Winter M, Wood JLN (2018) Bovine TB Strategy Review. October 2018 Defra, London, UK.

7. Department for Environment, Food and Rural Affairs (2011) Bovine Tuberculosis Eradication Programme for England, UK.

8. Department for Environment, Food and Rural Affairs (2014) The Strategy for achieving Officially Bovine Tuberculosis Free status for England, UK.

9. Department for Environment, Food and Rural Affairs (2017) Guidance to Natural England Licenses to kill or take badgers for the purpose of preventing the spread of bovine TB under section 10(2)(a) of the Protection of Badgers Act 1992.

10. Brunton LA, Donnelly CA, O'Connor H, Prosser A, Ashfield S, et al. (2017) Assessing the effects of the first 2 years of industry-led badger culling in England on the incidence of bovine tuberculosis in cattle in 2013-2015. Ecol Evol p. 1-18.

11. Bourne J, Donnelly C, Cox D, Gettinby G, McInerney J, et al. (2007) Bovine TB: The Scientific Evidence. A Science Base for a Sustainable Policy to Control TB in Cattle. Final Report of the Independent Scientific Group on Cattle TB presented to the Secretary of State for Environment, Food and Rural Affairs the Rt Hon David Miliband MP pp. 287.

12. Donnelly CA, Woodroffe R, Cox DR, Bourne FJ, Cheeseman CL, et al. (2006) Positive and negative effects of widespread badger culling on tuberculosis in cattle. Nature 439(7078): 843-846.

13. Donnelly CA, Wei G, Johnston WT, Cox DR, Woodroffe R, et al. (2007) Impacts of widespread badger culling on cattle tuberculosis: concluding analyses from a large-scale field trial. Int J Infect Dis 11(4): 300-308.
14. Krebs J, Anderson R, Clutton-Brock T, Morrison I, Young D, et al. (1997) Bovine tuberculosis in cattle and badgers: Report to The Rt Hon Dr Jack Cunningham MP, Ministry of Agriculture, Fisheries and Food pp. 191.

15. Donnelly CA, Hone J (2010) Is there an association between levels of bovine tuberculosis in cattle herds and badgers? Stat Commun Infect Dis 2: 1 .

16. Francis J (1947) Bovine Tuberculosis, Staple Press, London, UK.

17. Zuckerman S (1980) Badgers, Cattle and Tuberculosis. HMSO, London, UK.

18. Skuce RA, Allen AR, Mc Dowell SWJ (2011) Bovine tuberculosis (TB): a review of cattle to cattle transmission, risk factors and susceptibility. Bacteriology Branch. Veterinary Sciences Division Agri food and Biosciences Institute.

19. Vernon MC (2011) Demographics of cattle movements in the United Kingdom. BMC Veterinary Research 7: 31.

20. Amos W, Brooks-Pollock E, Blackwell R, Driscoll E, Nelson-Flower M, et al. (2013) Genetic Predisposition to Pass the Standard SICCT Test for Bovine Tuberculosis in British Cattle. Plosone 38(3): e58245.

21. Karolemeas K, Donnelly CA, Conlan AJK, Mitchell AP, Clifton-Hadley RS, et al. (2012) The Effect of Badger Culling on Breakdown Prolongation and Recurrence of Bovine Tuberculosis in Cattle Herds in Great Britain. Plosone 7(12): e51342.

22. Swift BMC, Huxley JN, Plain KM, Begg DJ, de Silva K, et al. (2016) Evaluation of the limitations and methods to improve rapid phage-based detection of viable Mycobacterium avium subsp. paratuberculosis in the blood of experimentally infected cattle. Veterinary Research 12: 115.

23. Animal Plant and Health Agency (2018) Exceptional private use of nonvalidated tests for TB on cattle in England.

24. Monaghan ML, Doherty ML, Collins JD, Kazda JF, Quinna PJ (1994) The tuberculin test Veterinary Microbiology 40(1-2): 111-124

25. Nunez-Garcia J, Downs SH, Parry JE, Abernethy DA, Broughan JM, et al. (2017) Meta-analyses of the sensitivity and specificity of ante-mortem and post-mortem diagnostic tests for bovine tuberculosis in the UK and Ireland. Prev Vet Med 1(153): 94-107.

26. de la Rua-Domenech R, Goodchild AT, Vordermeier M, Clifton-Hadley R (2006) Ante mortem diagnosis of Bovine Tuberculosis: the significance of unconfirmed test reactors. Government Veterinary Journal 16(1): 65-71.

27. de la Rua-Domenech R, Goodchild AT, Vordermeier HM, Hewinson RG, Christiansen KH, et al. (2006) Ante mortem diagnosis of tuberculosis in cattle: a review of tuberculin tests, y-interferon assay and other ancillary diagnostic techniques. Res Vet Sci. 81: 190-210.

28. Goodchild AV, Downs SH, Upton P, Wood JLN, de la Rua-Domenech R (2015) Specificity of the comparative skin test for bovine tuberculosis in Great Britain. Veterinary Record 177: 258.

29. More SJ, McGrath G (2015) Randomized Badger Culling Trial: interpreting the results. Veterinary Record.

30. Jenkins HE, Woodroffe R, Donnelly CA (2008) The effects of annual widespread badger culls on cattle tuberculosis following the cessation of culling. Int J Infect Dis 12(5): 457-465.

31. Jenkins HE, Woodroffe R, Donnelly CA (2010) The duration of the effects of repeated widespread badger culling on cattle tuberculosis following the cessation of culling. Plosone 5(2): e9090.

32. Donnelly CA (2013) Results from the Randomized Badger Culling Trial based on data downloaded in July 2013. Report to Defra by Imperial College London, UK. 
33. Donnelly CA, Nouvellet P (2013) The Contribution of Badgers to Confirmed Tuberculosis in Cattle in High-Incidence Areas in England. PLOS Currents Outbreaks.

34. King D (2007) Bovine tuberculosis in cattle and badgers. A report by the chief scientific advisor, Sir David King, Submitted to the Secretary of State, DEFRA, July 2007.
35. Woodroffe R, Donnelly CA, Cox DR, Bourne FJ, Cheeseman CL, et al. (2006) Effects of culling on badger Meles meles spatial organization: implications for the control of bovine tuberculosis. J Appl Ecol 43: 1-10.

36. Godfray HCJ, Donnelly CA, Kao RR, Macdonald DW, McDonald RA, et al. (2013) A restatement of the natural science evidence base relevant to the control of bovine tuberculosis in Great Britain. Proc R Soc Lond pp. 280.

\section{Your next submission with Juniper Publishers will reach you the below assets}

- Quality Editorial service

- Swift Peer Review

- Reprints availability

- E-prints Service

- Manuscript Podcast for convenient understanding

- Global attainment for your research

- Manuscript accessibility in different formats (Pdf, E-pub, Full Text, Audio)

- Unceasing customer service

Track the below URL for one-step submission https://juniperpublishers.com/online-submission.php 